\title{
Supporting Information for The Effect of Graft Density on The Ordering of Block Copolymer Grafted Nanoparticles
}

\author{
S M Al Islam Ovy, Joshua Obinwa, Andrew J. Peters* \\ College of Engineering \& Science, Louisiana Tech University, Ruston, LA 71272, USA \\ *e-mail: apeters@latech.edu
}




\section{i) Allowable Domain Size, Measurement of Domain Size \& Box Size}

Allowable domain size can be measured by following equation: ${ }^{1,2}$

$$
P=\frac{D_{x} D_{y} D_{z}}{\sqrt{\left(n_{x} \cdot D_{y} D_{z}\right)^{2}+\left(n_{y} \cdot D_{x} D_{z}\right)^{2}+\left(n_{z} \cdot D_{x} D_{y}\right)^{2}}}
$$

where $\mathrm{P}$ is an allowable domain size, $\mathrm{D}_{\mathrm{i}}$ refers to the box dimension in the $i$ direction, and $\mathrm{n}_{\mathrm{i}}$ is an integer greater than or equal to zero.

For a box size of 35 , the allowable domains greater than 10 are: $10.1036,10.5529,11.068$, 11.6667, 12.3744, 14.2887, 15.6525, 17.5, 20.2073, 24.7487, and 35. The measured domain sizes for our system and the method used to measure them are shown below. The difference between these allowable domains and any domain that may form is less than $10 \%$ for domains smaller than $\sim 27$ and is generally around $5 \%$ or less. The free energy penalty for a small deviation ( 10\%) from the optimal domain size is generally small. ${ }^{3}$ This can be seen in block copolymer directed self-assembly where block copolymers commonly adjust to patterns that are not perfectly commensurate to their preferred domain sizes. Often, variations of $10 \%$ or more are found to be acceptable and still form high quality lamellae. ${ }^{3-5}$ Additionally block copolymers will still form lamellae when non-commensurate with a guiding pattern, but defects will form; they do not disorder. Therefore, while there is some small amount of error that is present because the box size is not allowed to change (not possible for the given model) it is small, and would likely not produce a disordered state, but defective lamellae which could have been easily identified. 
In our calculation, the domain size was measured by calculating the structure factor $(\mathrm{S}(\mathrm{q}))$ using the Faber-Ziman method ${ }^{6-8}$ with a Lorch window function. ${ }^{9,10}$

$$
S(q)-1=4 \pi \rho_{0} \int_{0}^{\infty} r^{2} \frac{\sin q r}{q r}[g(r)-1] \frac{\sin \left(\pi r / r_{\max }\right)}{\pi r / r_{\max }} d r
$$

Here, $g(r)=$ The pair distribution function takes for $D$ beads with $D$ beads, and the domain size $D$ is calculated by,

$$
D=\frac{2 \pi}{q^{*}}
$$

Here, $q^{*}$ is the location of the maximum in $\mathrm{S}(\mathrm{q})$.

For large domain systems, the results consist of some error. To rectify the error a correction factor has been used. ${ }^{6}$

$$
\% \text { Error }=\frac{L_{\text {measured }}-L_{0}}{L_{0}} \times 100
$$

\section{ii) Relation among Graft density, Length of graft, Domain Size}

While the lamellar state is an energy minimum, the box size used here is constant and therefore only allows a finite number of repeat distances to occur without defect. Domain size calculated by using equations (2) \& (3). Domain sizes are shown in the lamellar state, except when a system never formed lamellae. Lamellae were not formed for graft density $0.4 / d^{2}$ because the system 
was kinetically trapped and experienced a significant slowdown in equilibration. So, for this particular graft density domain size was calculated from the disordered state.

Domain size has been calculated when graft density is variable and graft length is changing.

\begin{tabular}{|c|c|c|}
\hline Graft Density, $G$ & Graft Length & Domain Size \\
\hline $1.2 / d^{2}$ & 18 & 14.51 \\
\hline $1 / d^{2}$ & 22 & 15.26 \\
\hline $0.8 / d^{2}$ & 28 & 17.82 \\
\hline $0.6 / d^{2}$ & 36 & 21.15 \\
\hline $0.4 / d^{2 *}$ & 54 & 25.43 \\
\hline
\end{tabular}

Domain size has been calculated when graft density is constant, but graft length is changing.

\begin{tabular}{|c|c|c|}
\hline Graft Density, $G$ & Graft Length & Domain Size \\
\hline $1.2 / d^{2}$ & 18 & 14.51 \\
\hline $1 / d^{2}$ & 22 & 15.89 \\
\hline $0.8 / d^{2}$ & 28 & 16.83 \\
\hline $0.6 / d^{2}$ & 36 & 20.43 \\
\hline $0.4 / d^{2 *}$ & 54 & 26.17 \\
\hline
\end{tabular}

Domain size has been calculated when graft length is constant, but graft density is changing.

\begin{tabular}{|c|c|c|}
\hline Graft Density, $G$ & Graft Length & Domain Size \\
\hline $1.2 / d^{2}$ & 18 & 14.51 \\
\hline $1 / d^{2}$ & 18 & 14.76 \\
\hline $0.8 / d^{2}$ & 18 & 14.47 \\
\hline $0.6 / d^{2}$ & 18 & 14.49 \\
\hline $0.4 / d^{2 *}$ & 18 & 14.25 \\
\hline
\end{tabular}

* - Graft Density $0.4 / d^{2}$ did not form lamellae. It was taken from disordered state while other graft densities are taken from lamellae state. 


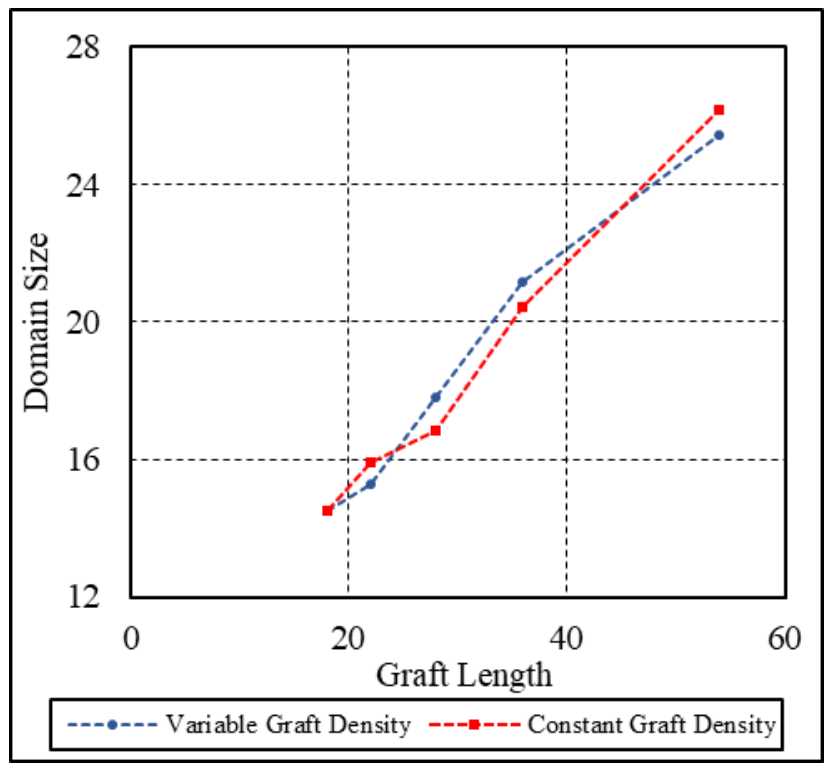

Figure S1 - Domain size as a function of graft length.

From Figure S1 it is clear that domain size mostly depends on graft length, not graft density.

\section{iii) Mean Square Displacement}

By calculating the mean square displacement (MSD) the diffusion of a particle can be measured.

We wanted to measure the diffusive timescale of the nanoparticle. For that reason, the DPD repulsive potential magnitudes were set to $A=25$ so there was no drive to phase separate. The distance from the particles original position was measured over time and distance were averaged over 10 independent runs. This average MSD is found in Figure S2 for different graft densities from $G=0.4 / d^{2}$ to $G=1.2 / d^{2}$. For each graft density a linear fit on MSD vs. time with the intercept set at the origin is taken and used to calculate the expected time to diffuse a given distance. This is plotted in Figure 1 and found to be 0.00004416 for graft density, $G=0.4 / d^{2}$ in units of distance $e^{2} /$ time. Domain size calculated for $G=0.4 / d^{2}$ is 12.6 and for a distance (d) of 
$12.6 / 4=3.15$ the diffusion time is 224,680 while for $d=12.6 / 2=6.3$ the diffusion time is 898,730 . The average time to diffuse D/4 is 152,770 timesteps for graft density $G=1.2 / d^{2}, 160,955$ timesteps for graft density $G=1.0 / d^{2}, 199,842$ timesteps for graft density $G=0.8 / d^{2}, 314,525$ timesteps for graft density $G=0.6 / d^{2}, 224,682$ timesteps for graft density $G=0.4 / d^{2}$.

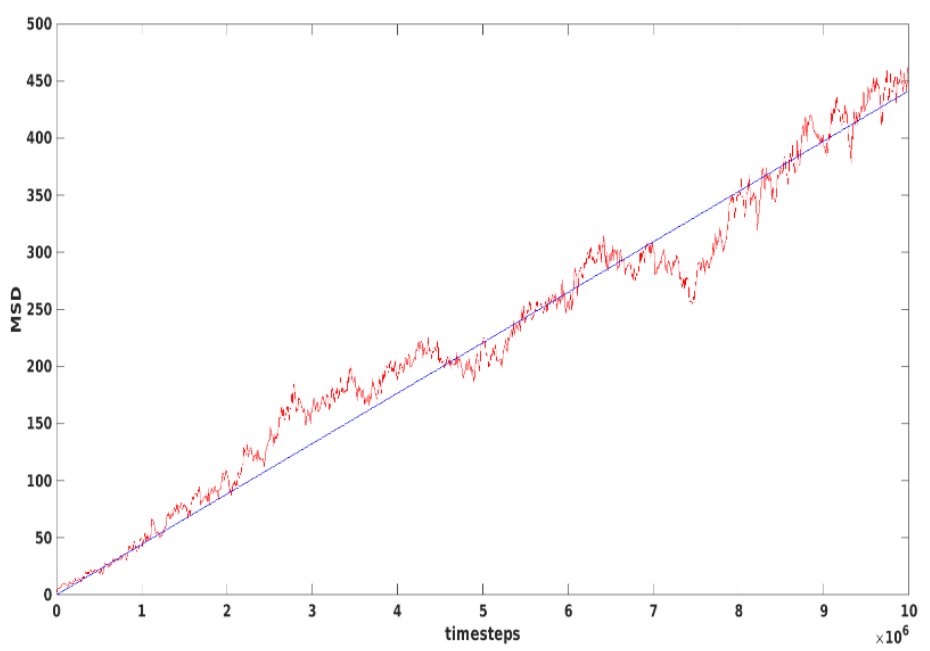

(a) $G=0.4 / d^{2}$

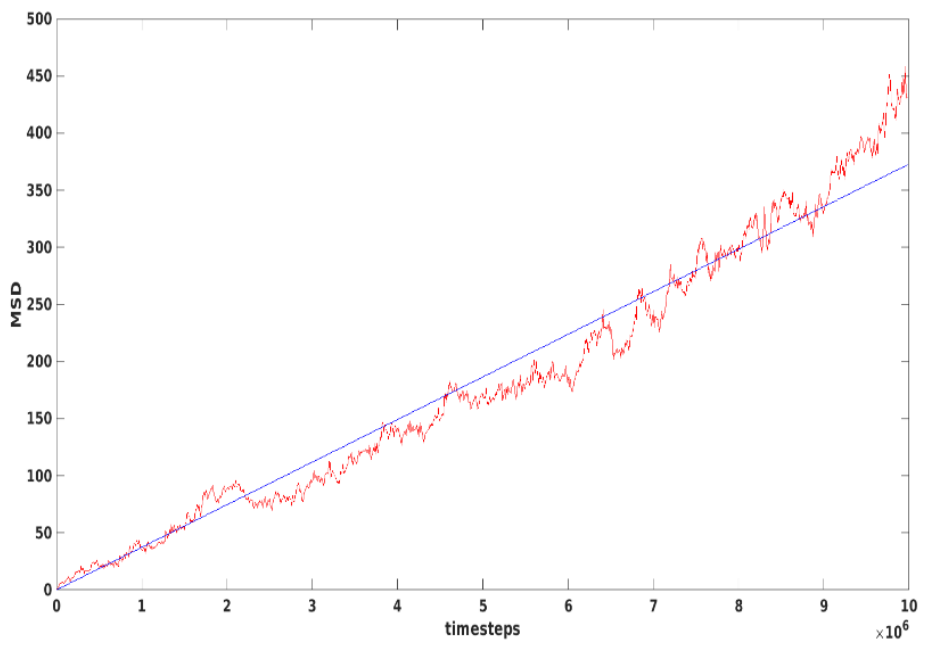

(b) $G=0.6 / d^{2}$ 


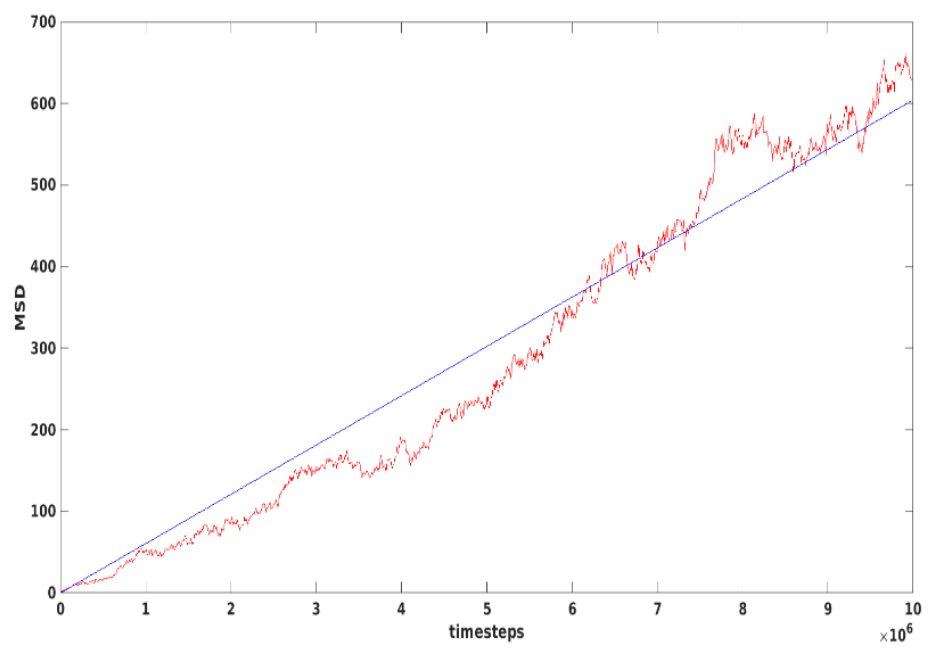

(c) $G=0.8 / d^{2}$

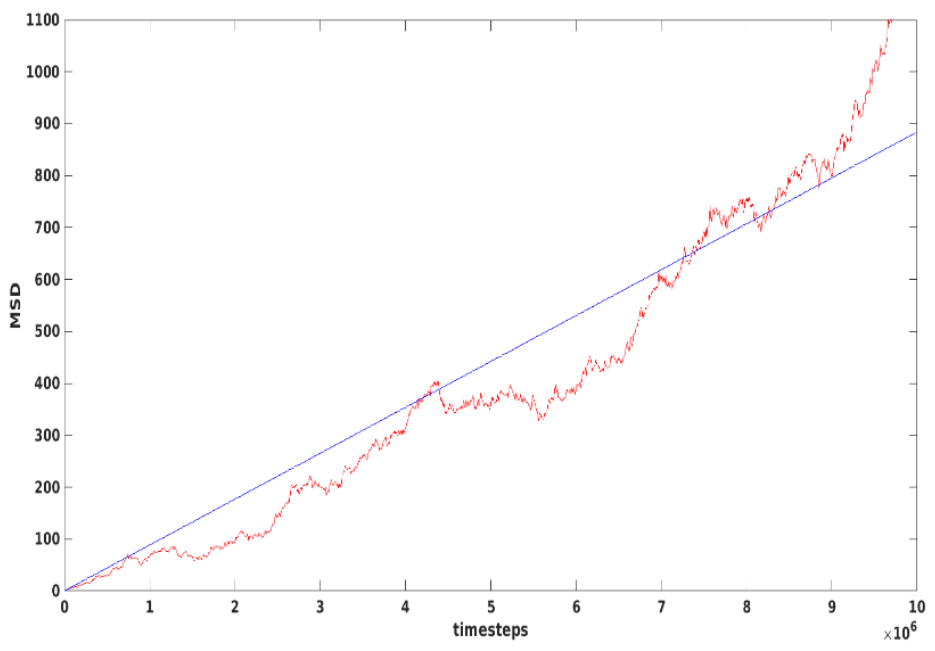

(d) $G=1.0 / d^{2}$ 


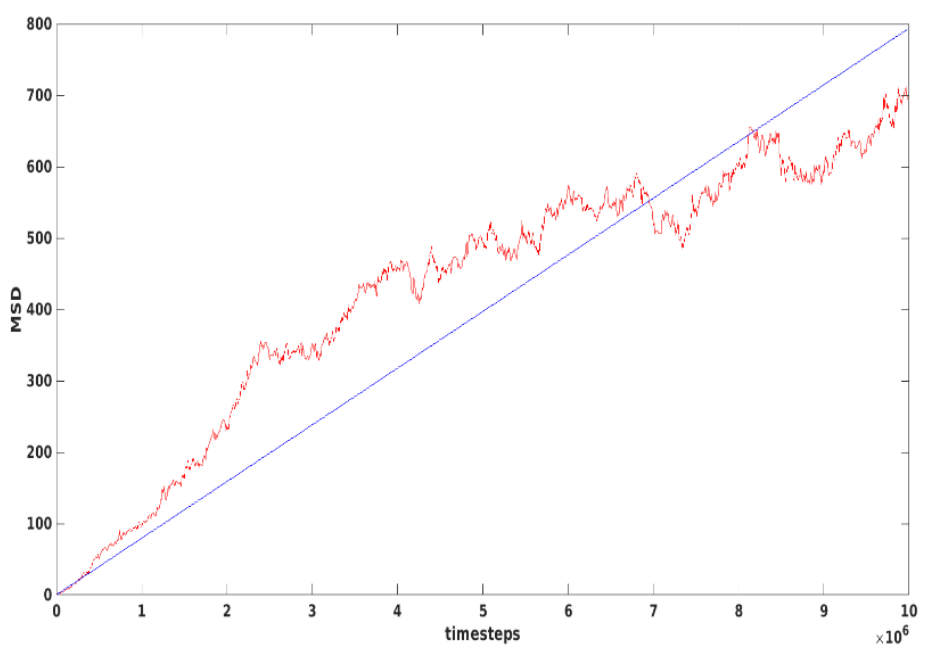

(e) $G=1.2 / d^{2}$

Figure S2 - Mean square displacement (MSD) of nanoparticle in polymer matrix at different graft density.

\section{iv) Particle Location and the Pair Distribution Function}

Particle location relative to the lamella centers and the pair distribution function between the nanoparticles were calculated as described in the methods section. Histograms of the centers of nanoparticles relative to the center of the domain are shown in Figure S3. Figures S4 and S5 show the pair distribution function $g(r)$ and a zoom in on the first peak respectively. The first peak represents particles that are in contact. Normalized particle count refers to the average number of particles in that bin per timestep. 

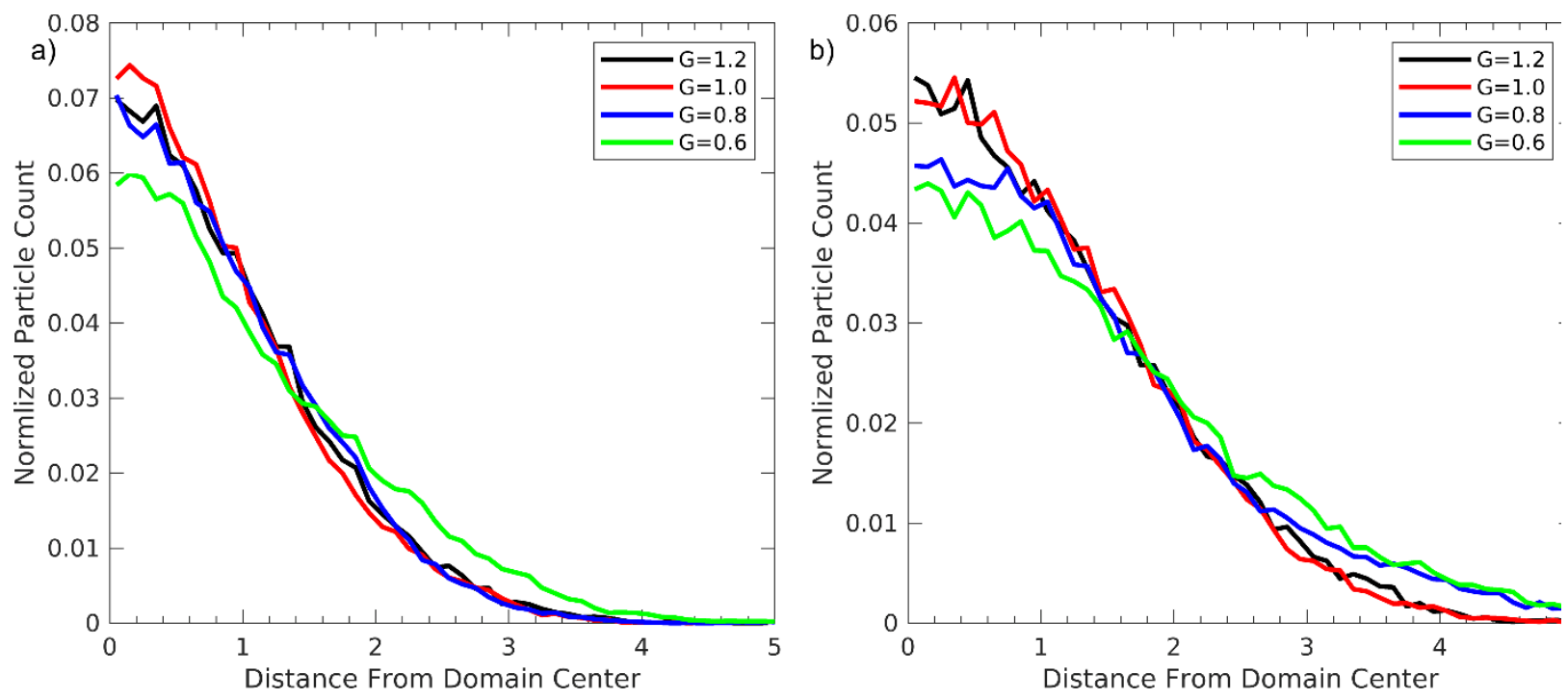

Figure S3. Histogram of particle centers for a) constant graft length, and b) constant polymer mass per particle. . a) shows constant graft length simulations, specifically $\xi=0.01$, except for $G=0.6$ for which $\xi=0.014$ was used because $\xi=0.01 \mathrm{did}$ not form lamellae. For $\mathrm{G}=0.6$ that forms lamellae, see Figure 11. b) shows constant polymer mass per particle simulations, specifically $\xi=0.008$. 

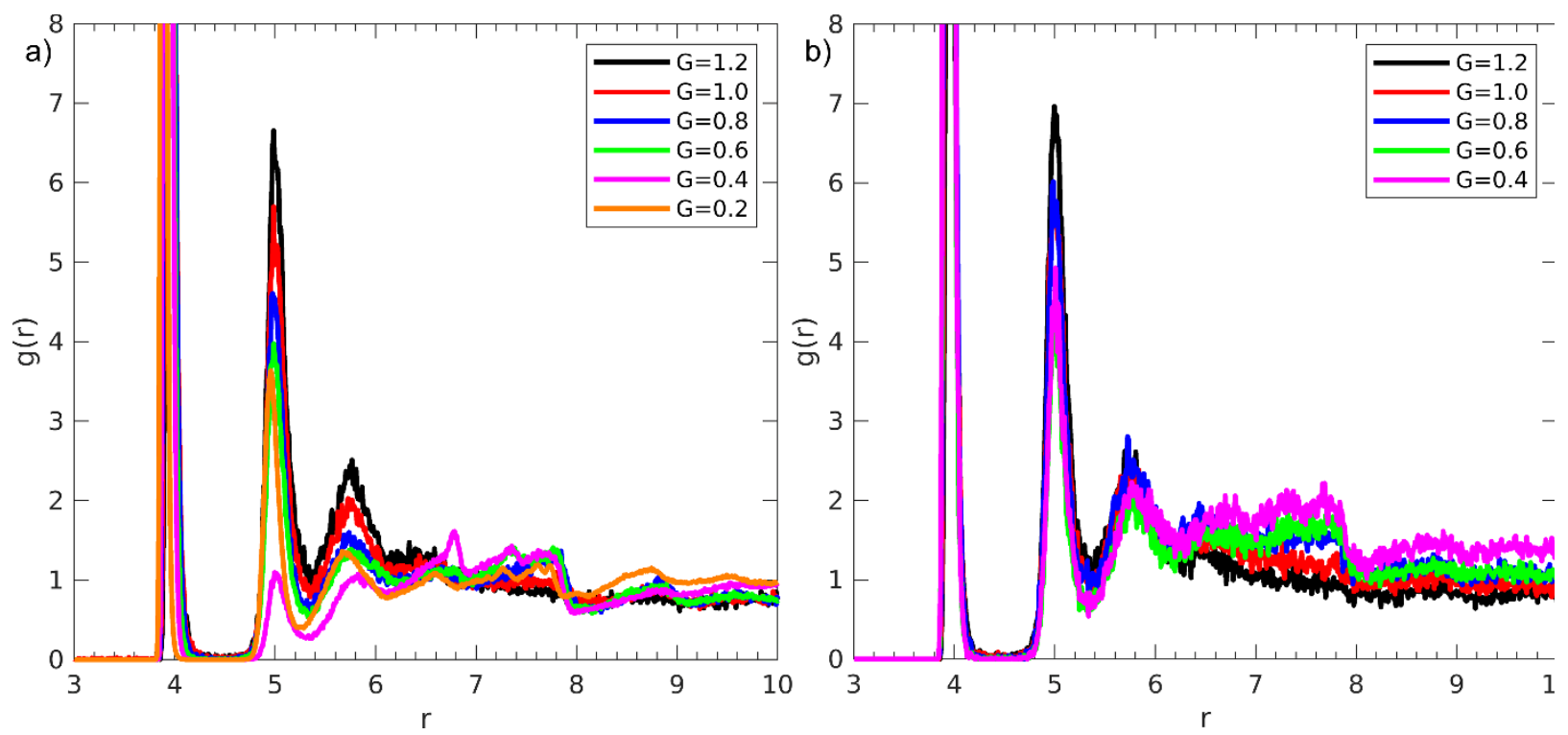

Figure S4. $g(r)$ between particles over the volume for a) constant graft length and b) constant polymer mass per particle simulations. The same simulations as for Figure 9 were used, that is, $\tau=1,000,000$ with $\xi=0.01$ for a) except for $\mathrm{G}=0.6,0.4$ or 0.2 for which $\xi=0.014$ was used, and $\xi=0.008$ for $b)$.
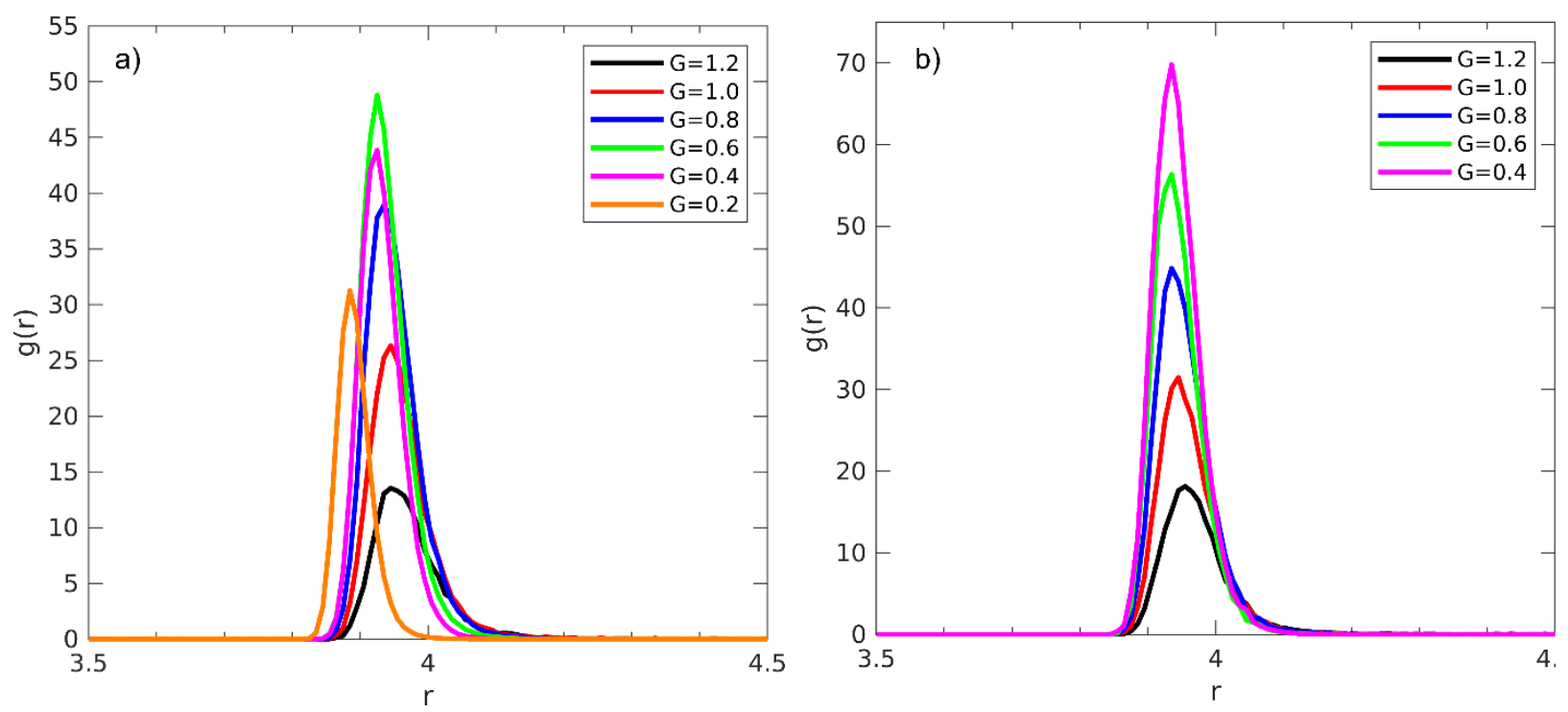

Figure S5. Zoom in of the first peak in Figure S4. 


\section{v) Effect of $\chi$ and $\xi$}

The repulsion parameter $A$ was varied for $\mathrm{G}=1.2$ and simulations were run for $\tau=1,000,000$. A decrease in $A$ corresponds to a decrease in $\chi$ and therefore drive to phase separation. A decrease in $\chi$ means that higher $\xi$ will disorder the system sooner since the magnitude of the energy barrier is determined by $\chi$. The same occurs for the minimum $\xi$. At some low $\chi$ no lamellae occur.

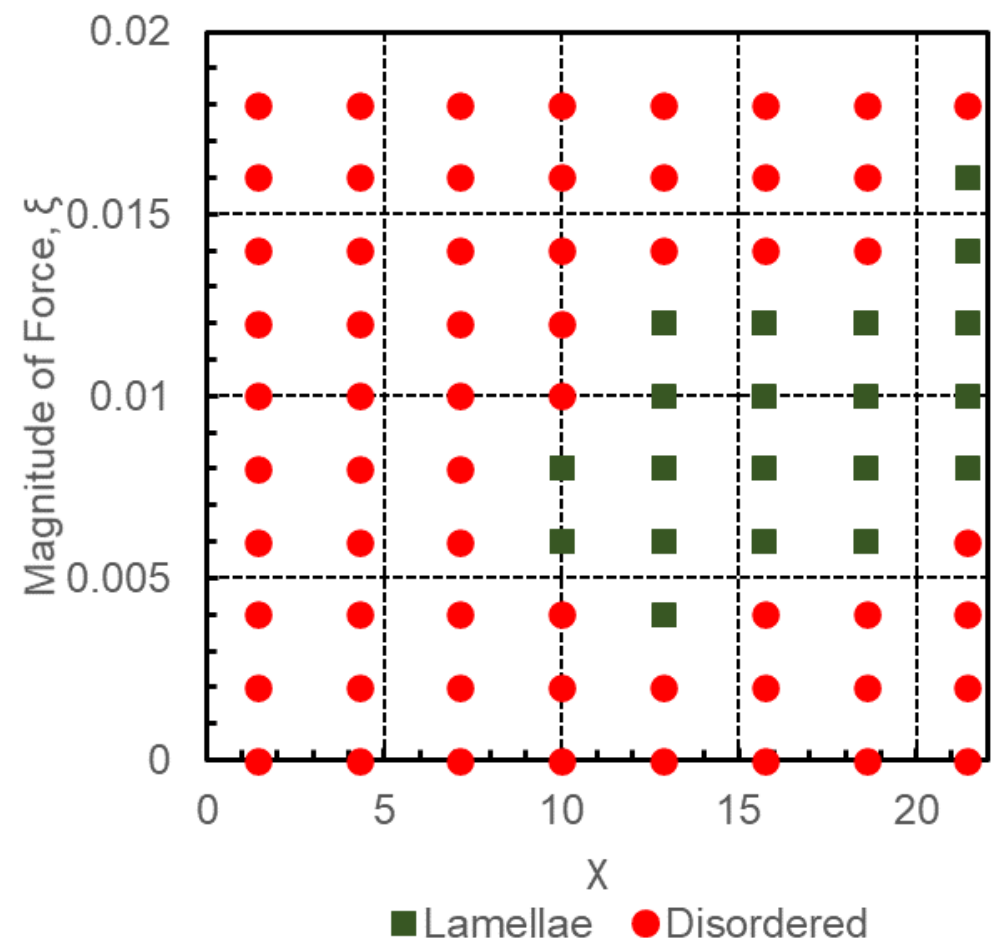

Figure S6. Lamellae vs. disordered results for $\mathrm{G}=1.2$ and $\tau=1,000,000$ when $\chi$ is varied.

\section{References}


(1) Peters, A. J. Mesoscale Simulation of Block Copolymer Phase Separation and Directed Self-Assembly Processes: Applications for Semiconductor Manufacturing. Georgia Institute of Technology 2015.

(2) Peters, A. J. Implementation and Optimization of Protracted Colored Noise Dynamics to Block Copolymer Grafted Nanoparticles. Comput. Mater. Sci. 2019, 167, 248-256.

(3) Peters, A. J.; Lawson, R. A.; Nation, B. D.; Ludovice, P. J.; Henderson, C. L. Free Energy Difference of Pitch Variation and Calculation of the Order-Disorder Transition in Block Copolymer Systems Using Thermodynamic Integration. Mater. Res. Express 2015, 2 (7), 75301.

(4) Gharbi, A.; Tiron, R.; Argoud, M.; Chevalier, X.; Barros, P. P.; Nicolet, C.; Navarro, C. Contact Holes Patterning by Directed Self-Assembly of Block Copolymers: Process Window Study. J. Micro/Nanolithography, MEMS, MOEMS 2015, 14 (2), 023508. https://doi.org/10.1117/1.jmm.14.2.023508.

(5) Cummins, C.; Kelly, R. A.; Gangnaik, A.; Georgiev, Y. M.; Petkov, N.; Holmes, J. D.; Morris, M. A. Solvent Vapor Annealing of Block Copolymers in Confined Topographies: Commensurability Considerations for Nanolithography. Macromol. Rapid Commun. 2015, 36 (8), 762-767. https://doi.org/10.1002/marc.201400722.

(6) Peters, A. J.; Lawson, R. A.; Nation, B. D.; Ludovice, P. J.; Henderson, C. L. CoarseGrained Molecular Dynamics Modeling of the Kinetics of Lamellar BCP Defect Annealing. Altern. Lithogr. Technol. VII 2015, 9423 (1), 94231Y. https://doi.org/10.1117/12.2085518.

(7) Faber, T. E.; Ziman, J. M. A Theory of the Electrical Properties of Liquid Metals: III. The 
Resistivity of Binary Alloys. Philos. Mag. 1965, 11 (109), 153-173.

(8) Fischer, H. E.; Barnes, A. C.; Salmon, P. S. Neutron and X-Ray Diffraction Studies of Liquids and Glasses. Reports Prog. Phys. 2005, 69 (1), 233.

(9) Lorch, E. Neutron Diffraction by Germania, Silica and Radiation-Damaged Silica Glasses. J. Phys. C Solid State Phys. 1969, 2 (2), 229.

(10) Du, J.; Benmore, C. J.; Corrales, R.; Hart, R. T.; Weber, J. K. R. A Molecular Dynamics Simulation Interpretation of Neutron and X-Ray Diffraction Measurements on Single Phase Y2O3-A12O3 Glasses. J. Phys. Condens. Matter 2009, 21 (20), 205102. 